To Appear in Astronomical Journal

Preprint typeset using $\mathrm{LAT}_{\mathrm{E}} \mathrm{X}$ style emulateapj v. 5/2/11

\title{
DISCOVERY OF X-RAY EMISSION IN THE OLD CLASSICAL NOVA DK LACERTAE
}

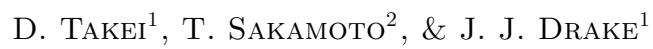

\begin{abstract}
We report the discovery of X-ray emission at the position of the old classical nova DK Lacertae using the Swift satellite. Three observations were conducted using the X-ray telescope 62 years after the discovery of the nova, yielding 46 source signals in an exposure time of $4.8 \mathrm{ks}$. A background-subtracted count rate was $9 \pm 2 \times 10^{-3}$ counts s$^{-1}$, corresponding to a detection significance level of $5 \sigma$. The X-ray spectrum was characterized by a continuum extending up to about $7 \mathrm{keV}$, which can be modeled by a power-law component with a photon index of $1.4-5.6$, or by a thermal bremsstrahlung component with a temperature of $0.7-13.3 \mathrm{keV}$, convolved with interstellar absorption with an equivalent hydrogen column density of $0.3-2.4 \times 10^{22} \mathrm{~cm}^{-2}$. Assuming a distance of $3900 \mathrm{pc}$ to the source, the luminosity was $10^{32}-10^{34} \mathrm{ergs} \mathrm{s}^{-1}$ in the $0.3-10 \mathrm{keV}$ energy band. The origin of X-rays is considered to be either mass accretion on the white dwarf or adiabatic shocks in nova ejecta, with the former appearing much more likely. In either case, DK Lacertae represents a rare addition to the exclusive club of X-ray emitting old novae.

Subject headings: novae, cataclysmic variables — stars: individual (DK Lacertae) - X-rays: stars
\end{abstract}

\section{INTRODUCTION}

A nova outburst is a cataclysmic nuclear explosion seen in the later stages of close binary star evolution. Such a close binary typically comprises a white dwarf and a red dwarf that fills its Roche lobe and transfers hydrogenrich gas to its companion. When the amount of accreted material reaches a critical mass, sudden hydrogen fusion is triggered by a thermonuclear runaway on the white dwarf surface (e.g., Starrfield et al. 2008). The released energy and material propagate through the circumstellar environment with typical ejecta velocities of the order of $1000 \mathrm{~km} \mathrm{~s}^{-1}$. The mass outflow continues until the nuclear fuel is consumed on the white dwarf, and the system then reverts back to a quiescent state with accompanying mass accretion. For extensive reviews of novae see e.g., Warner (2003); Bode \& Evans (2008).

Two different processes can be involved in producing $\mathrm{X}$-rays in quiescence. One of them is the release of gravitational energy through accretion of material onto the white dwarf (e.g., Hernanz \& Sala 2002), analogous to other cataclysmic variables (i.e., polars, intermediate polars, and dwarf novae). The other is through adiabatic shocks in the nova ejecta interacting with circumstellar environment, in analogy to a miniature supernova remnant in time and space scales (e.g., Balman 2005). In either case, X-ray detections of quiescent systems known to have undergone a nova explosion are quite rare. The new discoveries of X-ray emitting old novae provide us additional pieces to solve the puzzle of evolutionary channels in binaries followed by different kinds of cataclysmic variable activity.

Systematic X-ray studies of old novae are important for the purpose. However, existing wide-field X-ray surveys are not sensitive enough to detect emission from most

\footnotetext{
dtakei@head.cfa.harvard.edu

${ }^{1}$ Smithsonian Astrophysical Observatory, 60 Garden Street, Cambridge, MA 02138, US

2 Department of Physics and Mathematics, College of Science and Engineering, Aoyama Gakuin University, 5-10-1 Fuchinobe, Chuo-ku, Sagamihara-shi, Kanagawa 252-5258, Japan
}

objects in quiescence. Dedicated pointed observations are necessary, often with a high risk of detecting nothing. Here, the Swift observatory (Gehrels et al. 2004) provides an excellent opportunity to overcome the observational difficulties. Its X-ray telescope, the ability of the satellite to obtain snapshot observations with short exposures, and the Fill-in program to fill in gaps in the planned telescope schedule, provide an effective means for surveying potentially weak X-ray sources.

In this paper, we report the discovery of X-ray emission from the old classical nova DK Lacertae (hereafter DK Lac) for the first time. Using the Swift satellite, we detected a weak but significant X-ray signal 62 years after the optical discovery of its outburst. Despite the limited data with poor photon statistics, we further discuss these $\mathrm{X}$-ray properties and the nature of DK Lac.

\section{TARGET (DK LACERTAE)}

The classical nova DK Lac was discovered on 1950 January 23 at a brightness of 6 mag from photographic photometry (Bertaud 1950). A peak brightness of $5.8 \mathrm{mag}$ was also confirmed in data taken a day before the discovery (Bertaud \& Baldet 1952). Photometric and spectroscopic studies of the nova were subsequently conducted, which continued for about a year after the discovery (e.g., Gaposchkin 1957; McLaughlin 1950; Walker 1951; Wellmann 1951; Ribbe 1951; Pohl 1951; Bochníček 1951; Larsson-Leander 1953, 1954). The time evolution of the nova was moderately fast, with decline rates of $t_{2} \sim$ $19 \mathrm{~d}$ and $t_{3} \sim 32 \mathrm{~d}$, where $t_{2}$ and $t_{3}$ are the time to fade by 2 and $3 \mathrm{mag}$ from the optical maximum, respectively. Duerbeck (1981) derived a distance to the source of $1500 \pm 200 \mathrm{pc}$ based on a maximum magnitude versus rate-of-decline method.

DK Lac has been studied further in quiescence using ground-based telescopes. Henden et al. (2001) found a slow optical decline began in 2000 September (51 years after the discovery) for the first time, where the $V$-band magnitudes faded from 16.8 to $19.4 \mathrm{mag}$ by 2001 December (52 years after the discovery). Honevcutt et al. 
(2011) reported extensive photometric observations in 1990-2009 and witnessed a low brightness state by about $2 \mathrm{mag}$ in 2001-2003 (51-53 years after the discovery). Katysheva \& Shugarov (2007) found 0.1-0.2 mag oscillations with $0.1 \mathrm{~d}$ time-scales in $V$-band monitoring during 2003 (53 years after the discovery). The binary system was expected to have a mainly face-on inclination, with $i \lesssim 25-50 \mathrm{deg}$ based on $\mathrm{H} \alpha$ equivalent widths and $V$-band magnitudes (Honeycutt et al. 2011). The counterpart of the nova is located at (R.A., Decl.) = $\left(22^{\mathrm{h}} 49^{\mathrm{m}} 46^{\mathrm{s}} .91,+53^{\circ} 17^{\prime} 19^{\prime} \cdot 3\right)$ in the equinox J2000.0 with $0.3^{\prime \prime}$ position accuracy in the two micron all sky survey catalog (2MASS: Cutri et al. 2003).

A spatially-resolved nova remnant was first reported by Cohen (1985), based on a marginal detection in $6570 \AA$ and $\mathrm{H} \alpha$ images obtained in 1983-1984 (35 years after the discovery). The source radius was estimated to be $2^{\prime \prime}$ by stellar profile comparison. A remnant expansion velocity was found to be $1075 \mathrm{~km} \mathrm{~s}^{-1}$ from grating spectroscopy in 1984 August (Cohen 1985). Slavin et al. (1995) also confirmed the spatially extended emission in a $\mathrm{H} \alpha+[\mathrm{N}$ II] image $(6569 \AA)$ from 1993 September (44 years after the discovery), in which the remnant radius was $2.0-2.5^{\prime \prime}$. The distance to the source was revised to $3900 \pm 500 \mathrm{pc}$ based on nebular expansion parallax (Slavin et al. 1995). Gill \& O'Brien (2000) visited the system with the Wide Field Planetary Camera 2 onboard the Hubble Space Telescope in 1999 March 28 (49 years after the discovery), but no extended $\mathrm{H} \alpha+[\mathrm{N}$ II $]$ emitting remnant was detected. At that time, the $3 \sigma$ upper limit to the surface brightness was found to be $6 \times 10^{-16} \mathrm{erg} \mathrm{cm}^{-2} \mathrm{~s}^{-1} \operatorname{arcsec}^{-2}$.

\section{OBSERVATIONS AND REDUCTION}

We conducted a sequence of three pointing observations at the DK Lac position using the Swift observatory on 2012 April 13, 15, and 17 (Observation ID $=00045888001,00045888002$, and 00045888003, respectively). Swift has three instruments in operation (Gehrels et al. 2004): the Burst Alert Telescope (BAT: Barthelmy et al. 2005), the X-Ray Telescope (XRT: Burrows et al. 2005), and the Ultraviolet and Optical Telescope (UVOT: Roming et al. 2005). Here, we concentrate on the XRT data that show a significant X-ray detection at the nova position.

The XRT was operated in the state that selects the clocking mode automatically depending on source fluxes. The data presented here were mainly taken with Photon Counting mode, which provides full imaging and spectroscopic resolution with a frame time of $2.5 \mathrm{~s}$. For data reduction, we used the High Energy Astrophysics Software package version 6.11.1 and the calibration database version x20110725 and $\mathrm{m} 20120206$. The data were processed to level 2 event files with the standard screening criteria. The net total exposure time $\left(t_{\exp }\right)$ is $4.8 \mathrm{ks}$.

\section{ANALYSIS}

\subsection{Image Analysis}

At first data taken with the three Swift observations were merged. Figure 1 shows a smoothed image of the resulting XRT data in the $0.3-10 \mathrm{keV}$ energy band. An $\mathrm{X}$-ray source is clearly seen in this image. We estimated the source position using a two-dimensional fitting pro-

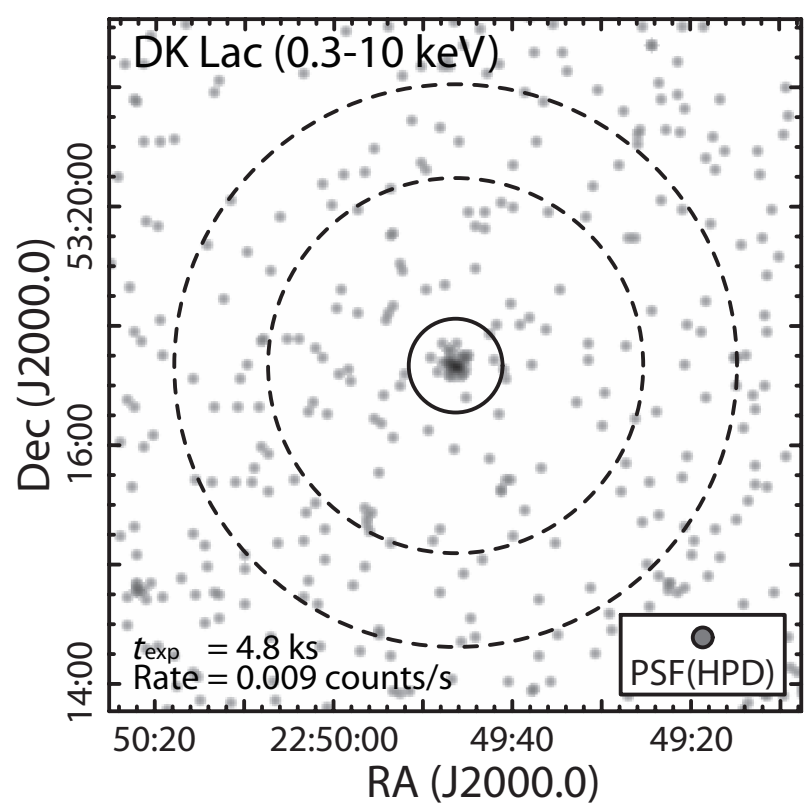

FIG. 1. - Smoothed XRT image in the 0.3-10 keV energy band. The source and background events for DK Lac were accumulated from the solid and dashed areas, respectively. The half-power size of the telescope PSF is shown in the bottom-right inset.

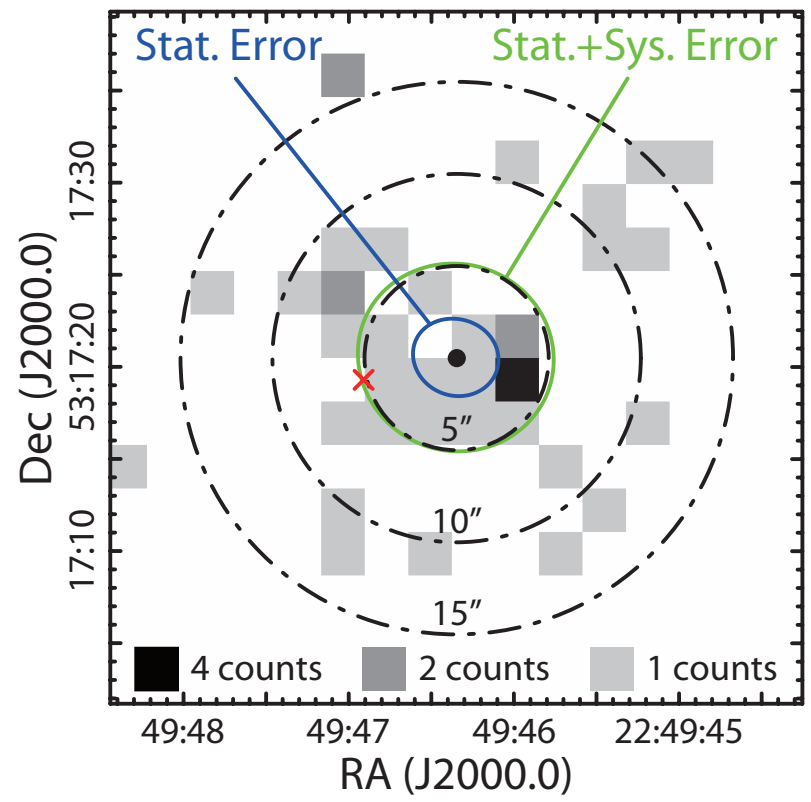

FIG. 2.- Close-up pixel image around DK Lac in the $0.3-10 \mathrm{keV}$ energy band. The 2MASS counterpart position is indicated by the red cross, while the estimated source position is shown by a black filled circle. The black dashed-and-dotted circles are distance indicators of $5^{\prime \prime}, 10^{\prime \prime}$, and $15^{\prime \prime}$ from the center. The blue circle is a statistical error region of an estimated position. The error region is expand to the green circle correcting for the nominal systematic uncertainty of the pointing accuracy of Swift.

cedure with the telescope point spread function (PSF). The radial profile of the XRT PSF can be numerically approximated by a King model, $\operatorname{PSF}(\mathrm{r})=\left(1+\left(r / r_{c}\right)^{2}\right)^{-\beta}$, where $r$ is a radial distance, $r_{c} \sim 5$.' 8 (2.46 pixels for the CCD detector) is a measure of the radial scale size, and $\beta \sim 1.55$ is a slope (Moretti et al. 2005). Events were taken from a circle with a radius of 20 pixels at positions derived using a recursive clipping algorithm. The 
Cash statistic (Cash 1979), a maximum likelihood function based on the Poisson distribution, was employed to find the best match between the model PSF and observed source count distribution. The best-fit position is (R. A., Decl. $)=\left(22^{\mathrm{h}} 49^{\mathrm{m}} 46.346,+53^{\circ} 17^{\prime} 20.48\right)$ with a $90 \%$ statistical confidence error of $\sim 2$ ". 3 (see black filled and blue open circles in Figure 2). The 2MASS position of DK Lac is located at a distance of $5^{\prime \prime} \cdot 2 \pm 00^{\prime \prime} 3$, which is consistent with the derived source position after allowing for the XRT pointing accuracy of $3^{\prime \prime}$ (Moretti et al. 2006).

A total of 46 source events were accumulated from a circle with a radius of 20 pixels, while 85 background events were taken from an annulus region with inner and outer radii of 80 and 120 pixels, respectively, at the best-fit position. The background region is about 20 times larger in area than that of the source, and the background-subtracted count rate was estimated to be $9 \pm 2 \times 10^{-3}$ counts s$^{-1}$ in the $4.8 \mathrm{ks}$ exposure time, corresponding to a detection significance of $5 \sigma$.

\subsection{Spatial Distribution}

In order to investigate the possibility that the XRT source has significant spatial extension, we computed the radial intensity profile at the derived best-fit position (Figure 31). The radial profile within a radius of 20 pixels $(47 ! 1)$ was further fitted by a normalized XRT PSF model (Moretti et al. 2005) and an additional background constant, yielding a null hypothesis probability close to unity and indicating statistical consistency with a point source. An upper limit to the X-ray source radius was estimated by two-dimensional image fitting using a simple disk model described by

$$
\operatorname{Disk}(\mathrm{r})=\left\{\begin{array}{cc}
A & \left(r \leq r_{\text {disk }}\right) \\
0 & \left(r>r_{\text {disk }}\right)
\end{array}[\text { counts }],\right.
$$

where an amplitude $(A)$ and a radius $\left(r_{\text {disk }}\right)$ of an emitting disk are treated as free parameters. The input source distribution was convolved with the telescope PSF function (Moretti et al. 2005), and an additional constant parameter was included in the fitting to represent background. The data were found to be consistent with a source radius smaller that $\sim 7^{\prime \prime}$, which corresponds to the $90 \%$ confidence level based on the Cash statistic (Figure (4). The best-fit radius is about $2^{\prime \prime}$, but is also statistically consistent with a radius of zero. The Swift data thus do not allow us to perform a definitive test of spatial extent, and are consistent with both a point-like nature and with significant extension of up to several arcseconds.

\subsection{Temporal and Spectral Analysis}

We examined the XRT light curve of the source using the same regions as in the above image analysis, and found the data to be consistent with the assumption of a constant flux. We then extracted the background-subtracted XRT spectrum, which is characterized by a continuum extending up to about $7 \mathrm{keV}$ (Figure 51). No discrete features were found either because of the poor photon statistics or because of the real spectrum. We fitted an unbinned spectrum in the $0.3-10 \mathrm{keV}$ energy band using the Cash statistic with a power-law or thermal bremsstrahlung component convolved with an interstellar absorption model (TBabs; Wilms et al. 2000). The best-fit values of power-law

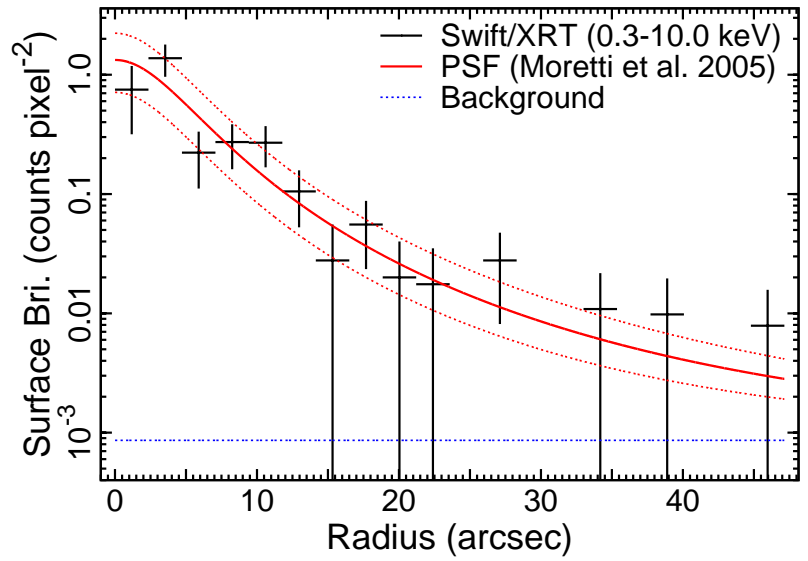

Fig. 3. - XRT radial profile of DK Lac. Data and $1 \sigma$ errors are shown with black crosses. The best-fit PSF model (Moretti et al. 2005) and the $1 \sigma$ confidence levels are shown with red solid and dashed lines, respectively. A background model is by the blue line.

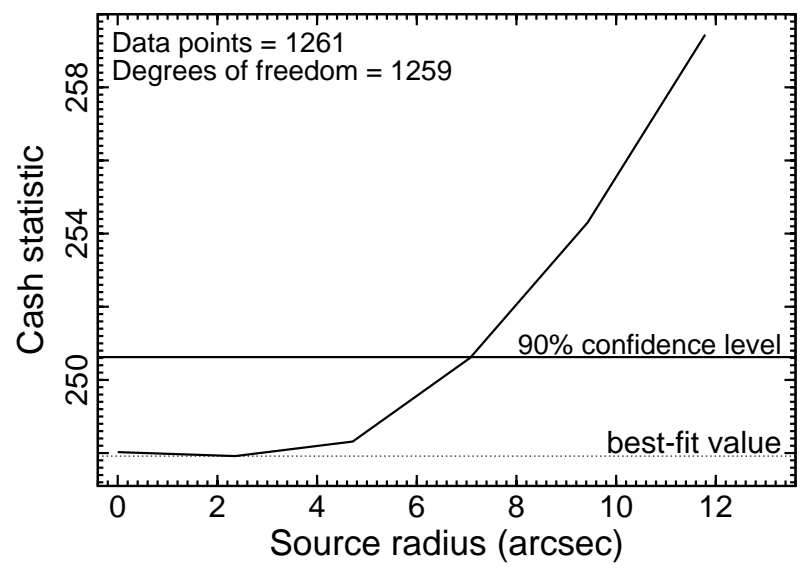

FIG. 4.- Confidence map of the two-dimensional image fitting to a flat disk model with different source radii. The vertical and horizontal axes are the Cash statistic value and the source radius, respectively. The two horizontal lines are the best-fit value and the upper boundary for the $90 \%$ confidence level, respectively.

photon index $(\Gamma)$, thermal bremsstrahlung temperature $\left(k_{\mathrm{B}} T\right)$, and a hydrogen equivalent column density $\left(N_{\mathrm{H}}\right)$ are summarized in Table 11. The source flux is $10^{-12}$ $10^{-14} \mathrm{ergs}^{-2} \mathrm{~s}^{-1}$ in the $0.3-10 \mathrm{keV}$ energy band, which corresponds to an absorption-corrected X-ray luminosity of about $10^{32}-10^{34} \mathrm{ergs} \mathrm{s}^{-1}$, assuming a distance of 3900 pc (Slavin et al. 1995).

The X-ray spectrum was also modeled using a cooling flow model originally developed for clusters of galaxies (Mushotzky \& Szymkowiak 1988), but also known to describe emission from some cataclysmic variables (Mukai et al. 2003; Pandel et al. 2005; Matranga et al. 2012) . In this model, the MEKAL code (Mewe et al. 1985) is employed to calculate the X-ray spectrum of optically-thin thermal plasma with a differential emission measure distribution corresponding to an isobaric radiatively cooling flow. Two dominant parameters, the higher plasma temperature $\left(k_{\mathrm{B}} T_{\mathrm{H}}\right)$ and the mass accretion rate $(\dot{M})$, were thawed for the spectral fitting, while the lower plasma temperature $\left(k_{\mathrm{B}} T_{\mathrm{L}}\right)$ and element abundances were fixed to $0.1 \mathrm{keV}$ and their solar values, respectively. In this model, the normalization of the mass accretion rate is also controlled by the redshift value 


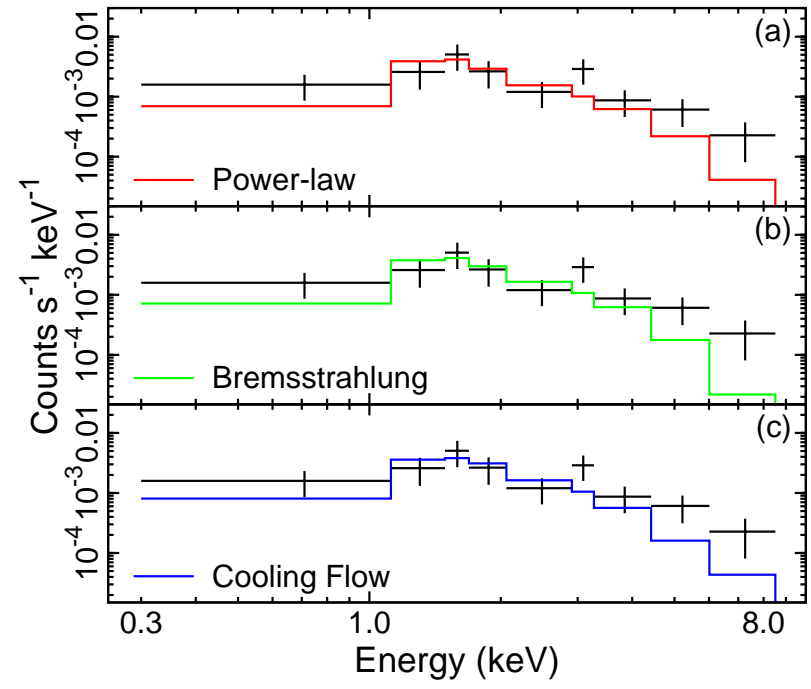

FIG. 5.- Background-subtracted XRT spectrum of DK Lac with the best-fit (a) power-law, (b) bremsstrahlung, and (c) cooling flow models. The data are shown as black crosses, while the best-fit models are represented color-coded by solid lines.

TABLE 1

Best-fit Parameters of the X-ray SPECTrum.

\begin{tabular}{|c|c|c|c|}
\hline Comp. & Par. & Unit & Value $^{a}$ \\
\hline Absorption & $N_{\mathrm{H}}$ & $\left(\mathrm{cm}^{-2}\right)$ & $1.1_{-0.8}^{+1.3} \times 10^{22}$ \\
\hline \multirow[t]{3}{*}{ Power-law } & $\Gamma$ & & $2.8_{-1.4}^{+2.8}$ \\
\hline & $F_{\mathrm{X}}^{\mathrm{b}}$ & $\left(\right.$ ergs s $\left.\mathrm{s}^{-1} \mathrm{~cm}^{-2}\right)$ & $3.0_{-2.4}^{+26.7} \times 10^{-13}$ \\
\hline & $L_{\mathrm{X}}^{\mathrm{b}, \mathrm{c}}$ & $\left(\operatorname{ergs~s}^{-1}\right)$ & $3.0_{-2.3}^{+25.9} \times 10^{33}$ \\
\hline Cash/d.o.f. & & & $224.38 / 966$ \\
\hline Absorption & $N_{\mathrm{H}}$ & $\left(\mathrm{cm}^{-2}\right)$ & $0.9_{-0.6}^{+0.9} \times 10^{22}$ \\
\hline \multirow[t]{3}{*}{ Bremsstrahlung } & $k_{\mathrm{B}} T$ & $(\mathrm{keV})$ & $2.0_{-1.3}^{+11.3}$ \\
\hline & $F_{\mathrm{X}}^{\mathrm{b}}$ & $\left(\operatorname{ergs~s}{ }^{-1} \mathrm{~cm}^{-2}\right)$ & $2.7_{-18}^{+15.0} \times 10^{-13}$ \\
\hline & $L_{\mathrm{X}}^{\mathrm{b}, \mathrm{c}}$ & $\left(\operatorname{ergs~s}^{-1}\right)$ & $1.3_{-0.8}^{+6.8} \times 10^{33}$ \\
\hline Cash/d.o.f. & & & $223.65 / 966$ \\
\hline Absorption & $N_{\mathrm{H}}$ & $\left(\mathrm{cm}^{-2}\right)$ & $1.4_{-0.7}^{+0.8} \times 10^{22}$ \\
\hline \multirow[t]{6}{*}{ Cooling Flow } & $k_{\mathrm{B}} T_{\mathrm{L}}$ & $(\mathrm{keV})$ & 0.1 (fixed) \\
\hline & $k_{\mathrm{B}} T_{\mathrm{H}}$ & $(\mathrm{keV})$ & $2.6_{-1.5}^{+8.0}$ \\
\hline & $z^{\mathrm{c}}$ & & $9.1 \times 10^{-7}$ (fixed) \\
\hline & $\dot{M}^{\mathrm{c}}$ & $\left(M_{\odot} \mathrm{yr}^{-1}\right)$ & $5.0_{-4.4}^{+39.8} \times 10^{-9}$ \\
\hline & $F_{\mathrm{X}}{ }^{\mathrm{b}}$ & $\left(\right.$ ergs s $\left.\mathrm{s}^{-1} \mathrm{~cm}^{-2}\right)$ & $2.8_{-2.5}^{+22.4} \times 10^{-13}$ \\
\hline & $L_{\mathrm{X}}{ }^{\mathrm{b}, \mathrm{c}}$ & $\left(\operatorname{ergs~s}^{-1}\right)$ & $2.2_{-2.0}^{+17.7} \times 10^{33}$ \\
\hline Cash/d.o.f. & & & $220.51 / 966$ \\
\hline
\end{tabular}

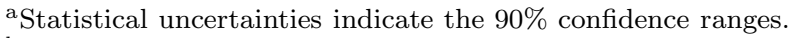

${ }^{\mathrm{b}}$ Values are in the $0.3-10 \mathrm{keV}$ energy band.

${ }^{\mathrm{c}}$ Values are for a distance of $3900 \mathrm{pc}$.

$(z)$ representing the source distance. We thus adopted $9.1 \times 10^{-7}$ for this parameter, corresponding to the distance to DK Lac of $3900 \mathrm{pc}$, assuming the Hubble constant of $70 \mathrm{~km} \mathrm{~s}^{-1} \mathrm{Mpc}^{-1}$ (e.g., Jarosik et al. 2011). The interstellar absorption (modeled using the TBabs model; Wilms et al. 2000) was also treated as a free parameter. The fitting results obtained with the cooling flow model are also summarized in Table 1

\section{DISCUSSION}

\subsection{Nature of the X-ray Source}

Based on astrometric consistency, the X-ray emission detected by the Swift XRT is considered to originate from the DK Lac system that underwent a classical nova explosion in 1950. We derived absorption with an equivalent hydrogen column density of $0.3-2.4 \times 10^{22} \mathrm{~cm}^{-2}$ along the line of sight to DK Lac. The values are consistent with or slightly higher than that of $3 \times 10^{21} \mathrm{~cm}^{-2}$ estimated from HI maps (Dickey \& Lockman 1990; Kalberla et al. 2005) for a $1^{\circ}$ cone radius around the DK Lac position. The absorption uncertainties depend on the adopted models, and the data preclude any indication of whether there might be excess absorption related to the circumstellar medium.

High energy emission in young and old novae can be divided into five known classes: (1) enigmatic non-thermal components (Takei et al. 2009; Abdo et al. 2010), (2) thermal plasma due to wind shocks in ejecta (e.g., Mukai \& Ishida 2001; Tsujimoto et al. 2009), (3) photospheric supersoft sources (e.g., Takei et al. 2008; Schwarz et al. 2011), (4) rekindled accretion (e.g., Hernanz \& Sala 2002; Takei et al. 2009), and (5) extended remnant emission (e.g., Balman 2005). The former three cases are typically seen during a nova event, and the remaining two in quiescence. The 1950 outburst of DK Lac was classified as a moderately fast nova, in which the event had almost terminated one year after its discovery (Bertaud \& Baldet 1952). Thus the origin of $\mathrm{X}$-rays 62 years after discovery is considered to be one of the latter cases noted above, i.e., due to rekindled accretion or nova remnant emission.

\subsection{Rekindled Accretion}

One of the favored scenarios for the X-ray emission in quiescence is accretion on the white dwarf. A nova event could temporary terminate the mass accretion process through destruction of an accretion disk during the blast, and by outward hydrodynamic pressure from a strong radiatively-driven wind (e.g. Drake \& Orlando 2010). The accretion process would then be expected to rejuvenate over time. Resumption of accretion soon after the blast has been suggested for e.g., the recurrent nova U Sco at 10 days after outburst (Mason et al. 2012), the classical nova V2491 Cyg at 50 days (Takei et al. 2009). Hernanz \& Sala (2002) found signs of accretion in V2487 Oph 2.7 years after that outburst. X-rays from rekindled accretion in classical novae also have been indicated for about 20 objects using the HEAO-1 satellite (Cordova et al. 1981), the ROSAT (Orio et al. 2001), and more recent observations with $A S C A, R X T E$, Suzaku, Chandra and XMM-Newton (e.g., Mukai \& Orio 2005; Orio et al. 2009). Thus, rekindled accretion in DK Lac 62 years after discovery would not be an unusual scenario. The large flickerings in optical brightness, presumably from accretion activity (e.g., Honevcutt et al. 2011), also support the interpretation that the process was rejuvenated.

For a nova explosion, the mass accretion rate of the binary system should be lower than $\sim 10^{-7} M_{\odot} \mathrm{yr}^{-1}$ to cause a thermonuclear runaway on a white dwarf surface without steady nuclear burning during accretion (Nomoto 1982). The derived accretion rate of $\lesssim 5 \times 10^{-8} M_{\odot} \mathrm{yr}^{-1}$ is small enough to engender a nova explosion. The time interval between nova eruptions is roughly in the range $10-10^{6} \mathrm{yr}$ or so, and mainly depends on the white dwarf mass and accretion rate (e.g., Yaron et al. 2005; Starrfield et al. 2008).

The nature of mass accretion in DK Lac depends on the magnetic field strength of the white dwarf. For nonmagnetic, or weakly magnetized, white dwarfs, an accre- 
tion disk is formed in the system, as in the dwarf nova case. For magnetized white dwarfs, the trajectory of infalling material is governed by the magnetic field, and an accretion funnel is formed around a magnetic pole on the white dwarf surface, as in the case of polars and intermediate polars (Cropper 1990; Patterson 1994). The estimated temperature of the X-ray emitting region of DK Lac $(\lesssim 10 \mathrm{keV})$ is slightly lower than the range of those in magnetized systems (e.g., Yuasa et al.|2010), but is similar to those in dwarf novae (e.g., Pandel et al. 2005; Ishida et al. 2009). The X-ray luminosities are also consistent with those in other non-magnetic or weakly magnetized cataclysmic variables (e.g., Baskill et al. 2005). Although the large uncertainties of these values do not allow us to deduce the magnetic type exactly, the $\mathrm{X}$ ray emission from DK Lac in quiescence represent most closely that expected from rekindled accretion in dwarf novae.

\subsection{Nova Remnant}

The other possible scenario is that X-ray emission originates from the surrounding nova remnant. The energy and material released in the nova explosion propagate through the circumstellar environment, and are expected to form a shock structure similar to those in supernova remnants. From this analogy, if we can observe nova remnants with sufficient sensitivity before the remnant energy has dissipated, we would naturally expect X-ray emission produced by adiabatic shocks.

$\mathrm{X}$-rays from nova remnants have only been reported in three objects to date: GK Per, RS Oph, and T Pyx. GK Per has the largest known remnant in solid angle with a radius of about $50^{\prime \prime}\left(4 \times 10^{12} \mathrm{~km}\right.$ at a distance of $470 \mathrm{pc}$ ), in which knots and clumps were spatially resolved in X-ray images obtained about 100 years after the outburst (Balman \& Ogelman 1999; Balman 2005). An X-ray nebular spectrum was observed, characterized by a combination of a non-equilibrium ionization thermal plasma model and a power-law component convolved with photoelectric absorption. The plasma temperature and photon index were approximately $1 \mathrm{keV}$ and 2 , respectively, and the X-ray luminosity was estimated to be $\sim 10^{31}-10^{33} \operatorname{ergs~s}^{-1}$ (Balman 2005). While GK Per is a system for which a nova outburst has been observed only once in a human time scale, T Pyx and RS Oph are known to show such outbursts recurrently. The Xray properties of $\mathrm{T}$ Pyx and $\mathrm{RS}$ Oph are otherwise fairly similar to those of GK Per: in all cases the presence of dense circumstellar material is expected to produce a high temperature plasma through strong shocks. The reported extended nebulae of $\mathrm{T}$ Pyx and $\mathrm{RS}$ Oph in Xrays were much more compact in solid angle than that of GK Per, with the radii of $\lesssim 10^{\prime \prime}$ (Luna et al. 2009; Balman 2010). In the case of T Pyx, Balman (2012) and Balman et al. (2012) find extension of less than $1^{\prime \prime}$ based on Chandra observations, though Montez et al. (2012) find no evidence for extended emission.

We have noted that the current XRT data do not allow us to make a definitive conclusion regarding the presence of an extended X-ray remnant for DK Lac. At face value, the best-fit X-ray source radius is $2^{\prime \prime}$ (Figure 4). This value corresponds to the size of the extended optical shell (Cohen 1985; Slavin et al. 1995), and also does not exceed the expected size of $\sim 3-4^{\prime \prime}$ at the epochs of our
$\mathrm{X}$-ray observations. It corresponds to a nebular radius of $1 \times 10^{12} \mathrm{~km}$ at a distance of $3900 \mathrm{pc}$, which is of the same order as that of GK Per. The estimated temperature and luminosity of DK Lac are similar to those of the other nova. If DK Lac has an extended X-ray remnant with a radius of a few arcsec, it could be confirmed with the higher spatial resolution of the Chandra X-ray Observatory.

There are, however, three opposing arguments to the extended remnant scenario for explaining the X-ray emission. (1) The difference in optical and X-ray luminosities: the upper limit of the optical flux 49 years after discovery was $6 \times 10^{-16} \mathrm{ergs} \mathrm{cm}^{-2} \mathrm{~s}^{-1} \operatorname{arcsec}^{-2}$ (Gill \& O'Brien 2000), which corresponds to an optical luminosity of $\sim 10^{30} \operatorname{ergs~s}^{-1} \operatorname{arcsec}^{-2}$ at a distance of 3900 pc. Even if the DK Lac nebular has a radius of $7^{\prime \prime}$ at maximum, the X-ray luminosity of $10^{32}-10^{34} \mathrm{ergs} \mathrm{s}^{-1}$ is slightly higher than that in the optical. This implies that the dominant nebular emission is in X-rays, which is not the case for other novae. (2) Currently, there is no clear evidence of radio emission - if DK Lac has a similar nebular to GK Per, the radio flux is expected to be about $0.3 \mathrm{mJy}$ in $1.5 \mathrm{GHz}$ at $3900 \mathrm{pc}$ from Seaquist et al. (1989). This is enough to be detectable but DK Lac was not observed in previous radio surveys (e.g., Bode et al. 1987). Future observations at radio wavelengths to search for this would be worthwhile. (3) There is no evidence of dense circumstellar material. The three known novae with extended X-ray nebulae are all rather different from typical classical novae. GK Per had a planetary nebula before the outburst (Seaquist et al. 1989), while T Pyx and RS Oph are recurrent novae that have substantial pre-outburst circumstellar material with which the ejecta can interact. Neither of these situations are the case for DK Lac. While we cannot conclude that a substantial X-ray remnant is not present, it is much more likely that rekindled accretion is responsible for the observed X-rays.

\section{CONCLUSION}

We have discovered X-ray emission from the old classical nova DK Lac, 62 years after its only known outburst. The emission originates either in mass accretion onto the white dwarf, or in adiabatic shocks in the nova remnant. The present data do not allow us to discriminate between these two scenarios, although rekindled accretion is more likely. Higher resolution X-ray imaging observations to resolve any extended X-ray emission and radio observations to search for emission resulting from circumstellar shocks would be of considerable interest. In either case, DK Lac would represent a rare addition to the currently very small sample of X-ray emitting old novae.

The authors thank the Swift principal investigator and operations team for allocating and scheduling the telescope time for our novae survey program. D. T. is financially supported by the Japan Society for the Promotion of Science. J. J. D. was supported by the NASA contract NAS8-39073 to the CXC and thanks the Director, H. Tananbaum, for continuing advice and support. Finally, we thank an anonymous referee for a very useful report that enabled us to significantly improve the manuscript. 


\section{REFERENCES}

Abdo, A. A., Ackermann, M., Ajello, M., et al. 2010, Science, 329, 817

Balman, S.. 2005, ApJ, 627, 933

Balman, Ş. 2010, MNRAS, 404, L26

Balman, S. 2012, ArXiv e-prints

Balman, Ş. \& Ögelman, H. B. 1999, ApJ, 518, L111

Balman, S., Starrfield, S., Drake, J. J., et al. 2012, The Astronomer's Telegram, 3915, 1

Barthelmy, S. D., Barbier, L. M., Cummings, J. R., et al. 2005, Space Science Reviews, 120, 143

Baskill, D. S., Wheatley, P. J., \& Osborne, J. P. 2005, MNRAS, 357,626

Bertaud, C. 1950, IAU Circ., 1254, 1

Bertaud, C. \& Baldet, F. 1952, Journal des Observateurs, 35, 108

Bochníček, Z. 1951, Bulletin of the Astronomical Institutes of Czechoslovakia, 2, 88

Bode, M. F. \& Evans, A. 2008, Classical Novae, ed. Bode, M. F. \& Evans, A. (Cambridge: Cambridge University Press)

Bode, M. F., Seaquist, E. R., \& Evans, A. 1987, MNRAS, 228, 217

Burrows, D. N., Hill, J. E., Nousek, J. A., et al. 2005, Space Science Reviews, 120, 165

Cash, W. 1979, ApJ, 228, 939

Cohen, J. G. 1985, ApJ, 292, 90

Cordova, F. A., Jensen, K. A., \& Nugent, J. J. 1981, MNRAS, 196, 1

Cropper, M. 1990, Space Sci. Rev., 54, 195

Cutri, R. M., Skrutskie, M. F., van Dyk, S., et al. 2003, VizieR Online Data Catalog, 2246, 0

Dickey, J. M. \& Lockman, F. J. 1990, ARA\&A, 28, 215

Drake, J. J. \& Orlando, S. 2010, ApJ, 720, L195

Duerbeck, H. W. 1981, PASP, 93, 165

Gaposchkin, C. H. P. 1957, The galactic novae.

Gehrels, N., Chincarini, G., Giommi, P., et al. 2004, ApJ, 611, 1005

Gill, C. D. \& O'Brien, T. J. 2000, MNRAS, 314, 175

Henden, A., Freeland, E., \& Honeycutt, K. 2001, IAU Circ., 7777, 3

Hernanz, M. \& Sala, G. 2002, Science, 298, 393

Honeycutt, R. K., Kafka, S., Jacobson, H., et al. 2011, AJ, 141, 122

Ishida, M., Okada, S., Hayashi, T., et al. 2009, PASJ, 61, 77

Jarosik, N., Bennett, C. L., Dunkley, J., et al. 2011, ApJS, 192, 14

Kalberla, P. M. W., Burton, W. B., Hartmann, D., et al. 2005, A\&A, 440, 775

Katysheva, N. A. \& Shugarov, S. Y. 2007, in Astronomical Society of the Pacific Conference Series, Vol. 372, 15th European Workshop on White Dwarfs, ed. R. Napiwotzki \& M. R. Burleigh, 523

Larsson-Leander, G. 1953, Stockholms Observatoriums Annaler, 17,8

Larsson-Leander, G. 1954, Stockholms Observatoriums Annaler, 18,3

Luna, G. J. M., Montez, R., Sokoloski, J. L., Mukai, K., \& Kastner, J. H. 2009, ApJ, 707, 1168

Mason, E., Ederoclite, A., Williams, R. E., Della Valle, M., \& Setiawan, J. 2012, A\&A, 544, A149
Matranga, M., Drake, J. J., Kashyap, V., \& Steeghs, D. 2012 , ApJ, 747, 132

McLaughlin, D. B. 1950, AJ, 55, 174

Mewe, R., Gronenschild, E. H. B. M., \& van den Oord, G. H. J. 1985, A\&AS, 62, 197

Montez, R., Sokoloski, J. L., \& Nelson, T. 2012, The Astronomer's Telegram, 4097, 1

Moretti, A., Campana, S., Mineo, T., et al. 2005, in Society of Photo-Optical Instrumentation Engineers (SPIE) Conference Series, Vol. 5898, Society of Photo-Optical Instrumentation Engineers (SPIE) Conference Series, ed. O. H. W. Siegmund, 360-368

Moretti, A., Perri, M., Capalbi, M., et al. 2006, A\&A, 448, L9

Mukai, K. \& Ishida, M. 2001, ApJ, 551, 1024

Mukai, K., Kinkhabwala, A., Peterson, J. R., Kahn, S. M., \& Paerels, F. 2003, ApJ, 586, L77

Mukai, K. \& Orio, M. 2005, ApJ, 622, 602

Mushotzky, R. F. \& Szymkowiak, A. E. 1988, in NATO ASIC Proc. 229: Cooling Flows in Clusters and Galaxies, ed. A. C. Fabian, 53-62

Nomoto, K. 1982, ApJ, 253, 798

Orio, M., Covington, J., \& Ögelman, H. 2001, A\&A, 373, 542

Orio, M., Mukai, K., Bianchini, A., de Martino, D., \& Howell, S. 2009, ApJ, 690, 1753

Pandel, D., Córdova, F. A., Mason, K. O., \& Priedhorsky, W. C. 2005, ApJ, 626, 396

Patterson, J. 1994, PASP, 106, 209

Pohl, E. 1951, Astronomische Nachrichten, 280, 139

Ribbe, J. 1951, PASP, 63, 39

Roming, P. W. A., Kennedy, T. E., Mason, K. O., et al. 2005, Space Science Reviews, 120, 95

Schwarz, G. J., Ness, J.-U., Osborne, J. P., et al. 2011, ApJS, 197, 31

Seaquist, E. R., Bode, M. F., Frail, D. A., et al. 1989, ApJ, 344, 805

Slavin, A. J., O’Brien, T. J., \& Dunlop, J. S. 1995, MNRAS, 276, 353

Starrfield, J. H., Illiadis, C., \& Hix, W. R. 2008, in Classical Novae, ed. M. Bode and A. Evans (Cambridge: Cambridge University Press), 77-101

Takei, D., Tsujimoto, M., Kitamoto, S., et al. 2008, PASJ, 60, 231

Takei, D., Tsujimoto, M., Kitamoto, S., et al. 2009, ApJ, 697, L54

Tsujimoto, M., Takei, D., Drake, J. J., Ness, J.-U., \& Kitamoto, S. 2009, PASJ, 61, S69

Walker, M. F. 1951, PASP, 63, 34

Warner, B. 2003, Cataclysmic Variable Stars, ed. Warner, B. (Cambridge: Cambridge University Press)

Wellmann, P. 1951, ZAp, 29, 112

Wilms, J., Allen, A., \& McCray, R. 2000, ApJ, 542, 914

Yaron, O., Prialnik, D., Shara, M. M., \& Kovetz, A. 2005, ApJ, 623,398

Yuasa, T., Nakazawa, K., Makishima, K., et al. 2010, A\&A, 520, A25 